\title{
Realizability as a kind of truth-making
}

\author{
Øystein Linnebo and Stewart Shapiro
}

December 6, 2017

\section{Background on potential infinity}

Although this paper is self-contained, it is part of a larger project concerning potentiality in mathematics. The first and simplest case is the traditional Aristotelian notion of potential infinity (see [10]). An issue much like that of truthmaking arises in our explication of one of the options for potential infinity, namely how to make sense of generalizations from that perspective. We use the traditional intuitionistic notion of realizability to resolve the issue, and to help settle the correct logic for one kind of potential infinity.

We begin with some highlights of our account(s) of potential infinity. From Aristotle until the nineteenth century, the vast majority of major philosophers and mathematicians rejected the notion of the actual infinite. They argued that the only sensible notion is that of potential infinity-at least for scientific or, later, non-theological purposes.

In Physics 3.6 (206a27-29), Aristotle wrote, "For generally the infinite is as follows: there is always another and another to be taken. And the thing taken will always be finite, but always different"(2o6a27-29). As Richard Sorabji [12] (322-3) puts it, for Aristotle, "infinity is an extended finitude" (see also [7], [8]).

This orientation towards the infinite was endorsed by mainstream mathematicians as late as Gauss [5], who in 1831 wrote: "I protest against the use of infinite magnitude as something completed, which is never permissible in mathematics. Infinity is merely a way of speaking."

A definitive change in mathematicians' orientation towards the infinite took place late in the nineteenth century, resulting in large part from pioneering work by Georg Cantor, who showed us how to make mathematical sense of completed infinite collections or sets, and how to assign a size or cardinal number to such sets. Cantor's theory of infinite sets and numbers proved so elegant, insightful, and useful for mathematical purposes that it was quickly assimilated into mathematical practice, where it came to serve an important foundational role.

From this point on, the only sustained opposition to the Cantorian conception of the actual infinite came from intuitionists and constructive mathematics. 
They maintained that the the existence of a set-or any other kind of mathematical object-requires an explicit specification or construction. It follows that there can be no room for the actual or completed infinite. We can, with some idealization, be said to be able to construct arbitrarily large finite sets. But as finite creatures, it is out of the question that we ever complete the construction of an infinite set. It follows, intuitionists maintain, that the only permissible notion of infinite is the potential one.

We are particularly interested in some logical questions concerning potential infinity. Inspired, perhaps, by its only recent defender, many philosophers and logicians believe there is a connection between potential infinity and intuitionistic logic. Others deny the existence of any such connection. After all, thinkers from Aristotle until Gauss rejected the actual infinite in favor of the potential, but never questioned the law of excluded middle.

This current state of uncertainty and confusion concerning potential infinity is manifested in some questions raised by the acclaimed logician and philosopher William Tait:

Both Hilbert and the early intuitionists have associated commitment to the actual infinite with the use of classical logic, so that, for example, the use of quantification over the integers combined with classical logic commits one to the set of integers as an actual infinity. I would like someone to explain why this is the same notion of actual infinity as Aristotle's.

We thus have the following questions:

1. If the natural numbers are merely potentially infinite, are we entitled to quantify over all of them using (at least) intuitionistic logic?

2. Does quantification over all the natural numbers with classical logic presuppose actual infinity?

Our accounts invoke a modal language. To use the heuristic of possible worlds, the idea is that, for the Aristotelian, each world is finite, but each world has "access"' to another world, one with more mathematical objects ${ }^{1}$

Conside, for example, the natural numbers. According to the Aristotelian, and the intuitionists, the sequence of natural numbers is merely potentially infinite. For the intuitionist, they are constructed one at a time. For both camps, we never have all of the natural numbers, existing at once so to speak. The orientation is represented as the conjunction of the following theses:

$$
\begin{aligned}
& \square \forall m \diamond \exists n \operatorname{SUCC}(m, n) \\
& \neg \diamond \forall m \exists n \operatorname{SucC}(m, n),
\end{aligned}
$$

where $\operatorname{SUCC}(m, n)$ says that $n$ is an immediate successor of $m$.

\footnotetext{
${ }^{1}$ This talk of possible worlds is, for us, only a heuristic. If we accepted the existence of possible worlds, there would be an (actual) infinity of them.
} 
To be sure, the language of mathematics is strictly non-modal. Thus, when the question of the appropriate logic for reasoning about potential infinity arises, it typically does so in the ordinary, non-modal language of arithmetic.

We thus need a translation to serve as a bridge connecting the non-modal language in which mathematics is ordinarily formulated with the modal language in which our analysis of potential infinity is developed. Suppose we adopt a translation $*$ from the non-modal language, say $L$, to the corresponding modal language, say $L^{\diamond}$. The question of the right logic of potential infinity is the question of which entailment relations obtain in $L$. To determine whether $\phi_{1}, \ldots, \phi_{n}$ entail $\psi$, we need to apply the translation and ask whether $\phi_{1}^{*}, \ldots, \phi_{n}^{*}$ entail $\psi^{*}$ in the relevant modal system.

This means that the right logic of potential infinity depends on two factors. First, the logic obviously depends on our modal analysis of potential infinity; in particular, on the modal logic that is used in this analysis. Included here is also the background logic for the modal language, whether it is classical or intuitionistic, or something else. Second, the logic depends on the bridge that we choose to connect the non-modal language $L$ of ordinary mathematics with the modal language $L^{\diamond}$ in which our analysis of potential infinity is given.

To summarize, the modal logic we use is S4.2, which has the S4 axioms together with

$$
\diamond \square p \rightarrow \square \diamond p .
$$

The logic is sound and complete for Kripke frames that are reflexive and transitive, and have the following "convergence" property:

When a world $w_{0}$ accesses two other worlds $w_{1}$ and $w_{2}$, there is another world $w_{3}$ that both of the aforementioned worlds access.

$$
\forall w_{0} \forall w_{1} \forall w_{2}\left(w_{0} \leq w_{1} \wedge w_{0} \leq w_{2} \rightarrow \exists w_{3}\left(w_{1} \leq w_{3} \wedge w_{2} \leq w_{3}\right)\right)
$$

We also adopt the converse Barcan formula, as consistent with the underlying assumptions:

$$
\diamond \square p \rightarrow \square \diamond p .
$$

The translation goes as follows:

- The connectives are translated homophonically.

- An existential quantifier in mathematics $(\exists x)$ is rendered as $(\diamond \exists x)$. The idea is that an ordinary mathematical statement in the form "there is a number $n$ such that ..." is to be rendered "it is possible to construct a number $n$ such that ...".

- A universal quantifier in mathematics $(\forall x)$ is rendered as $(\square \forall x)$. So an ordinary statement in the form "for all numbers $n, \ldots$ " is to be rendered, "necessarily, all numbers (whenever constructed), ...". 


\section{Mirroring}

We now state two thereoms that relate the modal framework to its non-modal counterpart, via the translation. One is where the background modal logic is classical, and the other is where it is intuitionistic. The results support the foregoing framework.

For each formula $\phi$ in the non-modal language $L$, let $\phi^{\triangleright}$ be the translation of $\phi$ (i.e., with a $\square$ before each universal quantifier and a $\diamond$ before each existential quantier) into the modal language $L^{\diamond}$.

Say that a formula $\phi$ in the modal language is stable if the necessitations of the universal closures of the following two conditionals hold:

$$
\phi \rightarrow \square \phi \quad \neg \phi \rightarrow \square \neg \phi
$$

Intuitively, a formula is stable just in case it never "changes its mind", in the sense that, if the formula is true (or false) of certain objects at some world, it remains true (or false) of these objects at all accessible worlds as well. We argue elsewhere (that, for the notion of potential infinity, atomic formulas should be stable ([9], [10]) . Here is our first result.

Let $\vdash$ be the relation of classical deducibility in a non-modal first-order language, and $\vdash^{\diamond}$ be deducibility in this language corresponding by $\vdash, \mathrm{S} 4.2$, and axioms asserting the stability of all atomic predicates of the language.

Theorem 1: Classical mirroring For any formulas $\phi_{1}, \ldots, \phi_{n}, \psi$, we have:

$$
\phi_{1}, \ldots, \phi_{n} \vdash \psi \text { iff } \quad \phi_{1}^{\diamond}, \ldots, \phi_{n}^{\diamond} \vdash^{\diamond} \psi^{\diamond} .
$$

See [9], Theorem 5.4.

Our second result is a counterpart of this for an intuitionistic modal language. As usual, say that a formula $\phi$ is decidable in a given (intuitionistic) theory if the universal closure of $\phi \vee \neg \phi$ is deducible in that theory. Let $\vdash_{\text {int }}$ be the relation of intuitionistic deducibility in a first-order language, and let $\vdash_{\text {int }}^{\diamond}$ be deducibility in the corresponding modal language by $\vdash_{\text {int }}, \mathrm{S} 4.2$, the stability axioms for all atomic predicates, and the decidability of all atomic formulas.

Theorem 2: Intuitionistic potentialist mirroring For any formulas $\phi_{1}, \ldots, \phi_{n}, \psi$, we have:

$$
\phi_{1}, \ldots, \phi_{n} \vdash_{\text {int }} \psi \text { iff } \phi_{1}^{\diamond}, \ldots, \phi_{n}^{\diamond} \vdash_{\text {int }}^{\diamond} \psi^{\diamond} .
$$

See [10], Appendix B.

These theorems have a simple moral. Suppose we are interested in logical relations between the modalized translations, in a classical (or intuitionistic) modal theory that includes S4.2 and the stability axioms (and the decidabiity of atomic formulas). Then we may delete all the modal operators and proceed by the ordinary non-modal logic. In particular, under the stated assumptions, the modalized quantifiers $\square \forall$ and $\diamond \exists$ behave logically just as ordinary quantifiers, 
except that they generalize across all (accessible) possible worlds rather than a single world.

We take it that this buttresses our choice of the potentialist translation as the bridge connecting actualist and potentialist theories. We also get quick answers to our two guiding questions. If the natural numbers are merely potentially infinite, we are entitled to quantify over all of them using (at least) intuitionistic logic.

More important perhaps, is an answer to the second question. Quantification over all the natural numbers with classical logic does not presuppose actual infinity. The first mirroring theorem shows that ordinary classical firstorder logic is validated-provided it is sanctioned in the modal language.

Consider an Aristotelian notion of potential infinity. We take it this notion is based on some form of metaphysical modality, which behaves classically. Given this and the fact that Aristotle does not seem to allow any exceptions to the Law of Excluded Middle, he-and all the thinkers he inspired-are entitled to take the logic of potential infinity to be classical.

So there is no direct connection between potential infinity, as such, and intuitionistic logic. Motivation for the latter comes from a further orientation toward the underlying modality.

\section{The modality}

Actualism about the infinite unreservedly accepts actual infinities, and thus finds no use for modal notions in mathematics (or at least no use when it comes to the infinity). Actualists maintain that the non-modal language of ordinary mathematics is already fully explicit and thus deny that we need a translation into some modal language. We take it that this is the dominant view today. Actualists typically accept classical logic when reasoning about the infinite and typically also accept all of classical mathematics.

Potentialism is the orientation that stands opposed to actualism. According to this orientation, the objects with which mathematics is concerned are generated successively, and some of these generative processes cannot be completed.

Potentialists differ with respect to a qualitative matter. As characterized here, potentialism is the view that the objects with which mathematics is concerned are successively generated and that some of these generative processes cannot be completed. But what about the truths of mathematics? On any form of potentialism, these are modal truths concerned with certain generative processes. But how should these truths be understood?

Liberal potentialists regard the modal truths as unproblematic. In particular, there are modal truths about generative processes in their entirety, including those that cannot be completed.

Consider the Goldbach conjecture. As potentialists interpret it, the conjecture says that necessarily any even natural number that is generated can be written as a sum of two primes. Liberal potentialists maintain that this modal question has an unproblematic answer-it is either true or false. 
This approach to modal theorizing in mathematics is thus much like a realist approach to theorizing in general: there are objective truths about the relevant aspects of reality-modal reality in the case at hand. For our liberal potentialist, this objectivity warrants the use of some classical form of modal logic. Theorem 1 above, our first mirroring result, shows that, provided the modal logic is sufficiently strong, liberal potentialists are entitled to classical first-order logic in their ordinary mathematical reasoning about potential infinity.

The matter is closely related to the so-called weak Brouwerian counterexamples to excluded middle. The liberal potentialist insists that there will or will not be a sequence of twelve consecutive 5 's somewhere in the decimal expansion of $\pi$. This is not, of course, because the sequence exists all at once, so to speak, as an actual infinity. Rather, the liberal potentialist notes that, in this case, the digits of the sequence are completely determined by a fixed rule, and it is this rule that guarantees that either there will or will not be the run of 5's.

Ian Rumfitt [11], §7.4, argues against the intuitionistic use of weak counterexamples (not under that name) against classical logic. If the decimal expansion of $\pi$ does contain the pattern in question, then, of course, that will be discovered eventually (idealizing, of course). Rumfitt argues that if "the pattern occurs nowhere in the expansion, it will lie in the rule for expaning $\pi$, together with the axioms that characterize the sequence of natural numbers, that it occurs nowhere" (p. 204). Rumfitt thus articulates and supports what we call liberal potentialism here ${ }^{2}$

The strict potentialist goes beyond the liberal view by requiring, not only that every object be generated at some stage of a process, but also that every truth be "made true" at some stage. For example, "18 is the sum of two primes" is plausibly taken to be "made true" once the numbers up through 18 have been generated. By that stage, we have established that $5+13=18$ and $7+11=18$. There is no need to look beyond these five numbers to determine the truth of our example.

\section{Generality}

For the strict potentialist, an existential generalization is plausibly "made true" once a witness is generated, and shown to be a witness. That is, a sentence in the form $\exists x \Phi(x)$ is made true once a number $n$ is generated where it is made true that $\Phi(n)$. This much does not seem particularly problematic.

By contrast, universal generalizations over the natural numbers pose a problem for strict potentialism. Again, consider Goldbach's conjecture. Since the conjecture is concerned with all the natural numbers-whenever they may be

\footnotetext{
${ }^{2}$ Since our liberal potentialist is a realist (in truth-value) about the modality, we might be tempted to call her a "modal realist", except, of course, that that label has already been taken. As noed, none of our characters is a Lewis-style realist about possible worlds, as that would require an actual infinity of worlds.
} 
generated-it is hard to see how it could be "made true" at any finite stage where, after all, only finitely many numbers will have been generated.

Our goal here is to provide a modest resolution of the issue, at least for the theory of the natural numbers, using some well-known work on intuitionistic systems.

If we are serious about the merely potential character of the sequence of natural numbers, and if we are strict about the modality, then there cannot be any arithmetical truths that are "made true" only by the sequence in its entirety.

All potentialists agree that when a sequence is incompletable, there is no such thing as "the sequence in its entirety". In short, the extra demand that differentiates strict from liberal potentialism puts great pressure on universal generalizations over a potentially infinite domain. To put it baldly, it is hard to see how a generalization over all $F^{\prime}$ s could be "made true" at some particular stage at which most $F^{\prime}$ s haven't even been generated.

For strict potentialism to provide a coherent conception of potential infinity, two challenges need to be met. First, the loose talk about being "made true" needs to be made formally precise. For example, we need to explain what it is for Goldbach's conjecture to be "made true" at some finite stage, despite the conjecture's concern with numbers not generated by that stage.

Second, we need to explain how strict potentialists can make sense of universal generalizations over potentially infinite collections, such as the natural numbers. Without the ability to state and prove such generalizations, we do not have a conception of the infinite, only a draconian restriction of quantificational reasoning to finite domains.

Our aim in what follows is to show how both challenges can be met and to show that this implies that the logic of potential infinity is intuitionistic, a more nuanced answer to our second guiding question. In particular, on our account, there is no guarantee that either the conjecture or its negation will be made true by any finite stage - thus the connection to excluded middle and intuitionistic logic.

What might it be for a universal generalization over the natural numbers to be "made true" at some finite stage? Traditional intuitionism, as well as many of its contemporary defenders, provide one answer, namely to identify mathematical truth with proof, or at least with algorithmic strategies guaranteed to yield proofs.

On this strong anti-realist conception of truth, a generalization is "made true" when we produce a proof of it. And since every proof is produced at some finite stage, this satisfies the strict potentialist's requirement. The problem is the strong anti-realism on which this answer rests. The generative process is understood as a process of actual constructions, whereby mathematical objects and truths/proofs-which did not previously exist or obtain-are brought into being.

It is better, we think, to not rely on this anti-realism. Fortunately, another route is available, one that avoids saddling strict potentialism with the controversial anti-realist views of traditional intuitionism. We take our cue from 
Hermann Weyl $([14], 54)$, who writes in a discussion of whether there is a natural number that has some decidable property $P$ as follows.

Only the finding that has actually occurred of a determinate number with the property $P$ can give a justification for the answer "Yes," and-since I cannot run a test through all numbers-only the insight, that it lies in the essence of number to have the property not$P$, can give a justification for the answer "No"; Even for God no other ground for decision is available.

On this view, the truth of the universal generalization - that every number is not- $P$ - has nothing to do with epistemic matters, such as proofs. Even God, who is assumed to know all the facts, cannot know facts that require running through all the natural numbers. The point is rather that, for our strict potentialist, there are no such facts.

Weyl's proposal, as we understand it, is that not every generalization is "made true" by the totality of its instances. When a universal generalization is true, it is instead made true by its lying in the essence number to have the relevant property.

By way of analogy, consider the truths that every red object is colored and that every atom of gold consists of 79 protons. These truths seem unconcerned with individual red objects or atoms of gold. They seem to be "made true" not by their instances but by what it is to be red or colored or gold. Likewise, we propose, there are essence-based constraints on any future generation of the objects studied by mathematics. For example, it is a constraint on the generation of natural numbers that the arithmetical successor operation be injective:

$$
\operatorname{Succ}\left(x, x^{\prime}\right) \wedge \operatorname{SucC}\left(y, y^{\prime}\right) \wedge x^{\prime}=y^{\prime} \rightarrow x=y
$$

This important arithmetical axiom is "made true" by the mentioned constraint prior to the generation of any particular natural numbers, or at least prior to the generation of all numbers.

Admittedly, these are deep metaphysical waters, even if not exactly those of orthodox intuitionism. In what follows, we explain the ideas in question and provide at least one precise mathematical model.

A striking feature of our explication of strict potentialism is that it leads to intuitionistic logic. This means that we provide a route from strict potentialism to intuitionistic logic that is independent of any form of anti-realism.

\section{A mathematical model of truthmaking}

In the case of arithmetic, at least, a good first approximation is provided by the realizability interpretation, going back to Stephen Cole Kleene [6], in 1945. The loose talk about what "lies in the essence of number" is to be understood in terms of (codes of) computable functions. 
For natural numbers $m, n$, let $[m, n]$ be the code of the ordered pair of $m$ and $n$. And let $\{e\}(n)$ be the result of applying Turing machine with index $e$ to the input $\bar{n}$. More formally, let $T(e, x, y)$ be the Kleene predicate, stating that $y$ is the code of a complete computation of the Turing machine with index $e$ and input $\bar{n}$. And if $y$ is the code of a complete computation, then let $U(y)$ be its output. Then the statement that $\{e\}(n)$ is defined is

$$
\exists y T(e, n, y) .
$$

And $\{e\}(n)=m$ comes to

$$
\exists y(T(e, n, y) \wedge m=U(y)) .
$$

We now define what it is for a natural number $e$ to be a realizer for a sentence $\phi$, written $e \Vdash \phi$. The idea is that $e$ encodes information that establishes the truth of $\phi$. For present purposes, a useful metaphysical heuristic is that $e$ functions as a "truth maker" for $\phi$.

- Atomic sentences are made true by computations, and so are unproblematic. The custom is to let any natural number realize any true atomic sentence (and no natural number realizes a false atomic sentence).

- A number $n$ realizes a sentence in the form $\phi \wedge \psi$ just in case $n$ is the code of an ordered pair whose first element reallizes $\phi$ and whose second element realizes $\psi$.

- A number $n$ realizes a sentence in the form $\phi \vee \psi$ just in case $n$ is the code of an ordered pair whose first element is 0 and whose second element reallizes $\phi$, or else $n$ is the code of an ordered pair whose first element is 1 and whose second element realizes $\psi$.

- A number $e$ realizes a sentence in the form $\phi \rightarrow \psi$ just in case for all numbers $n$, if $n$ realizes $\phi$, then $\{e\}(n)$ is defined, and realizes $\psi$.

- A number $n$ realizes a sentence in the form $\neg \phi$ just in case there is no number $m$ that realizes $\phi$.

- A number $n$ realizes a sentence in the form $(\exists x) \phi(x)$ just in case $n$ is $[p, q]$ and $p$ realizes $\phi(q)$.

For present purpooses, the most important clause is the one for the universal quantifier:

- $e$ realizes a sentence in the form $\forall x \phi(x)$ just in case for all $n,\{e\}(n)$ is defined and realizes $\phi(n)$.

That is, $e$ realizes the universal generalization $\forall n \phi(n)$ just in case the Turing machine $\{e\}$ computes a realizer for the instance $\phi(n)$ when given any numeral $\bar{n}$ as input. 
In terms of our metaphysical heuristic: $e$ is a truth maker for $\forall n \phi(n)$ just in case $e$ specifies a function that maps any numeral $\bar{n}$ to a truth maker for the associated instance $\phi(n)$.

Let's now regard a formula as true just in case it has a realizer or "truth maker". Since a realizer is just a natural number, this means that any true formula is made true after finitely many steps-once its realizer is generated. So our strict potentialist avoids having to wait until the end of time for at least some formulas to be made true.

Of course, there remains the question of whether this definition yields the right truths. For our strict potentialist, a natural measure of what is "right" is provided by the standard intuitionist theory of arithmetic, known as Heyting arithmetic, whose axioms as the same as those of first-order Peano-Dedekind arithmetic but where the underlying logic is intuitionistic, not classical.

Pleasingly, there is a rather straightforward theorem stating that Heyting arithmetic is sound with respect to the notion of truth that we have defined:

Theorem 3 (Realizability). Every theorem of Heyting arithmetic has a realizer.

However, there are theorems of first-order classical Dedekind Peano arithmetic that do not have a realizer. Indeed, there are sentences that have realizers that are classical logical falsehoods.

In this context, Church's thesis is the following scheme:

$$
\forall x \exists ! y \Phi(x, y) \rightarrow \exists e \forall x \exists z(T(e, x, z) \wedge \phi(x, U(z))),
$$

one instance for each formula $\Phi$ not containing $e$ free. In words, an instance of Church's thesis says that if, for each $x$ there is a unique $y$ such that $\Phi(x, y)$, then there is a Turing machine that computes the $y$ from the $x^{3}$

It is straightforward to show that every instance of Church's thesis has a realizer. But some instances of Church's thesis are provably false in classical arithmetic. Indeed, let $\Phi(x, y)$ be $[(y=1 \wedge \exists z T(x, x, z)) \vee(y=0 \wedge$ $\neg \exists z T(x, x, z)))]$. It follows from excluded middle that $\forall x \exists ! y \Phi(x, y)$, but, of course, no recursive function can compute this "self-halting" problem.

A. S. Troelstra [13], §1.11, shows how to formulate realizability within the language of arithmetic, and gives a sharp result. $\mathrm{HA}^{*}$ is a conservative extension of Heyting arithmetic, augmented with a term for the application of partial recursive functions, and $\mathrm{ECT}_{0}$ is a slight extension of Church's thesis. Then $\mathrm{HA}^{*}$ plus $\mathrm{ECT}_{0}$ entails that any formula $A$ is equivalent to the statement that $A$ has a realizer. So all we are entitled to assume concerning truth making at a world is intuitionistic logic (augmented with Church's thesis).

Let us now kick away the ladder of this talk about worlds in favor of just the modal operators, representing the generative modality and based on S4.2.

\footnotetext{
${ }^{3}$ The connection with the more usual, informal formulation of Church's thesis is that, on the so-alled BHK reading of the intuitionistic quantifiers, a formula in the form of the antecedent, $\forall x \exists ! y \Phi(x, y)$, means something like "given any $x$, one can effectively find a unique $y$ such that $\Phi(x, y)$. And the consequent is that there is a Turing machine that computes this value.
} 
It follows that this logic must be based on intuitionistic logic, not classical. Thus, for the strict potentialist, the appropriate modal system is one based on intuitionistic S4.2.

As before, the next step is to apply the potentialist translation to provide a bridge back to the non-modal language of ordinary mathematics. The result of doing so has already been established by Theorem 2, on intuitionistic potentialist mirroring. As we recall, the theorem ensures the following:

$$
\phi_{1}, \ldots, \phi_{n} \vdash_{\text {int }} \psi \text { iff } \quad \phi_{1}^{\diamond}, \ldots, \phi_{n}^{\diamond} \vdash_{\text {int }}^{\diamond} \psi^{\diamond} .
$$

This confirms a claim by Dummett and others, which has so far never been properly substantiated, namely that only intuitionistic quantification, not classical, is permitted over a domain that is potentially infinite (or "indefinitely extensible") - provided the potentiality is strict.

\section{Limitations, and future work}

These results, as pleasing as they are, do not readily extend to other, richer mathematical domains. For one thing, recall that Theorem 2, on intuitionistic mirroring, requires that atomic formulas are all decidable. This is an easy theorem for arithmetic, but it fails with the real numbers (where identity is not decidable).

Second, our account of truthmaking, via realizability, requires that each object in the domain-each natural number in the case at hand-has a canonical name. This, too, fails once we go beyond the natural numbers.

And third, our account uses recursive function theory, a rich and well developed account of computation over natural numbers and other finitary things like strings. There is interesting body of work on computations involving objects richer than natural numbers or strings. And there is an interesting body of work on realizabilty for richer intuitionistic theories, such as real analysis and set theory. But it is not at all clear, at this stage, whether this work can be used to provide an account of generality in those contexts.

\section{References}

[1] Aristotle, The Basic Works of Aristotle, R. McKeon, ed. (Random House, 1941)

[2] Cantor, G., Grundlagen einer allgemeinen Mannigfaltigkeitslehre. Ein mathematisch-philosophischer Versuch in der Lehre des Unendlichen (Leipzig, Teubner, 1883).

[3] Cantor, G., "Mitteilugen zur Lehre vom Transfinitten 1, II"., Zeitschrift für Philosophie und philosophische Kritik 91 (1887), 81-125, 252-270; 92, 250-265; reprinted in [4], 378-439. 
[4] Cantor, G., Gesammelte Abhandlungen mathematischen und philosophischen Inhalts, edited by E. Zermelo (Berlin, Springer, 1932).

[5] Gauss, Karl Friedrich, "Briefwechsel mit Schumacher", Werke, Band 8, 216 (1831).

[6] Kleene, Stephen Cole, "On the interpretation of intuitionistic number theory", Journal of Symbolic Logic 10 (1945): 109-124.

[7] Lear, Jonathan "Aristotelian infinity", Proceedings of the Aristotelian Society, new series 80 (1980), 187-210.

[8] Lear, Jonathan, "Aristotle's philosophy of mathematics", Philosophical Review 41 (1982), 161-192.

[9] Linnebo, Øystein, "The potential hierarchy of sets", Review of Symbolic Logic 6 (2013): 205-228.

[10] Linnebo, Øystein, and Stewart Shapiro, "Actual and potential infinity", Noûs, forthcoming.

[11] Rumfitt, Ian, The boundary stones of thought: an essay in the philosophy of logic, Oxford, Oxford University Press,

[12] Sorabji, Richard, Time, creation and the continuum: theories in antiquity and the early middle ages (Chicago, University of Chicago Press, 2006).

[13] Troelstra, A. S., "Realizability", in Handbook of proof theory: Studies in logic and the foundations of mathematics 137, edited by Samuel R. Buss, Amsterdam, North Holland Publishing Company, 1998, pp. 407-473.

[14] Weyl, Hermann, "Über die neue Grundlagenkrise der Mathematik", Mathematische Zeitschrift 10 (1921): 39-79. 